\title{
Primer registro de Scleroderma texense (Boletales, Sclerodermatinae) como una especie comestible en la Sierra Sur de Oaxaca, México
}

\author{
First record of Scleroderma texense \\ (Boletales, Sclerodermatinae) as an edible species \\ in the Sierra Sur from Oaxaca, Mexico
}

\begin{abstract}
Alonso Cortés-Pérez ${ }^{1}$, Cesar Kevin Pérez-Pacheco ${ }^{2}$, Elvia Yescas-Arreola ${ }^{3}$, Virginia Ramírez-Cruz ${ }^{4}$
1 Doctorado en Biosistemática, Ecología y Manejo de Recursos Naturales y Agrícolas, Universidad de Guadalajara, Apdo. Postal 1-139, Zapopan, C.P. 45101, Jalisco, México. ${ }^{2}$ Prolongación de Benito Juárez s.n., Barrio San Miguel, San Bartolomé Zogocho, C.P. 68857, Oaxaca, México. ${ }^{3}$ Bachillerato Integral Comunitario N ํ 9, Carretera a Santa María Zaniza s.n., Santiago Xochiltepec, Santiago Textitlán, C.P. 71342, Oaxaca, México. ${ }^{4}$ CONACyT-Departamento de Botánica y Zoología, Universidad de Guadalajara, Apdo. Postal 1-139, Zapopan, C.P. 45101, Jalisco, México.
\end{abstract}

\section{RESUMEN}

Antecedentes: Los hongos ectomicorrizógenos del género Scleroderma tienen distribución mundial, en la literatura se registran mayormente como tóxicos; aunque también se les atribuyen propiedades antiinflamatorias y hemostáticas. En México se conocen 14 especies, una de ellas, Scleroderma laeve está registrada como comestible de Hueyapan en el estado de Morelos.

Objetivo: Documentar el uso comestible de Scleroderma texense, así como algunos aspectos sobre la manera de cocinar el hongo por los zapotecos de Santiago Xochiltepec, Santiago Textitlán, Oaxaca.

Métodos: Se documentó la información mediante entrevistas informales y semiestructuradas aplicadas por estudiantes del Bachillerato Integral Comunitario $N^{\circ} 9$ de Santiago Xochiltepec como parte de un trabajo de investigación sobre hongos silvestres. Los especímenes se estudiaron con las técnicas empleadas en Micología.

Resultados y conclusiones: Se registra por primera vez Scleroderma texense como una especie comestible, este hongo es consumido por los zapotecos de Santiago Xochiltepec en la Sierra Sur del estado de Oaxaca. En esta localidad recibe el nombre de "huevo de pato", "huevo de guajolota" o "guitarra". Este registro contribuye al conocimiento sobre los usos de los hongos por los grupos étnicos de Oaxaca.

Palabras clave: etnomicología, hongo comestible, Sclerodermataceae

\section{ABSTRACT}

Background: The species included in Scleroderma have a worldwide distribution and are ectomycorrhizal, the genus has been recorded as toxic, although anti-inflammatory and hemostatic properties have been attributed to some species. In Mexico there are 14 species, one of them, Scleroderma laeve has been recorded as edible from Hueyapan in Morelos State.

Objective: To record the edible use of Scleroderma texense, as well as to explain how this mushroom is cooked by the Zapotecs from Santiago Xochiltepec, Santiago Textitlán, Oaxaca.

Methods: The information was documented through informal and semistructured interviews applied by high school students from Bachillerato Integral Comunitario $\mathrm{N}^{\circ} 9$ as a research project about mushrooms. The specimens were studied with the techniques used in Mycology.

Results and conclusions: Scleroderma texense is recorded for the first time as an edible species by the Zapotecs from Santiago Xochiltepec in the Southern Sierra of the State of Oaxaca. In this town it receives the name of "duck egg", "turkey egg" or "guitar". This record contributes to the knowledge about the uses of mushrooms by the ethnic groups in Oaxaca.

Keywords: ethnomycology, edible mushroom, Sclerodermataceae

\section{ARTICLE HISTORY}

Received 28 November 2020 / Accepted 26 June 2021

On line 16 July 2021
CORRESPONDING AUTOR

\. Virginia Ramírez-Cruz, virginia.ramirez@conacyt.mx

ORCID: 0000-0002-5197-1304 


\section{INTRODUCCIÓN}

El género Scleroderma Pers. pertenece a la familia Sclerodermataceae dentro del orden Boletales y agrupa de 30 a 46 especies a nivel mundial (Kirk et al. 2008; Guzmán et al. 2012; He et al. 2019). Las especies se distribuyen en regiones templadas, subtropicales y tropicales formando ectomicorrizas. En México, Guzmán et al. (2013) registraron la presencia de 14 taxones. Además de la gran importancia ecológica del género Scleroderma por sus relaciones simbióticas, existen ciertos registros de su uso comestible en el mundo y en México. Murrill (1910) anotó que comió $S$. citrinum Pers., sin ningún problema, pero que no resultó un hongo apetitoso; Sebek (1953) citó que las fases juveniles de varias especies de Scleroderma se emplean como adulterantes de trufas o incluso son de uso común en la Republica Checa. Así mismo Zhang et al. (2013) describieron Scleroderma yunnanense Y. Wang de Yunnan, China, como una especie endémica que es muy recolectada y vendida en los mercados populares de esa región y además considerada un hongo de buen sabor. En México, De Ávila et al. (1980) citaron a S. laeve Lloyd como un hongo comestible de Hueyapan, Morelos, México. Sin embargo, otros autores consideran a las especies del género como tóxicas (Coccia et al. 1990; Guzmán 1970, 1977, 1980). Existen casos de intoxicación transitorias gastrointestinales documentadas, por ejemplo, Stevenson y Benjamín (1961) describieron un caso provocado por $S$. cepa Pers. mientras que Guzmán (1970) citó una intoxicación ocurrida en Canadá por esta misma especie y otra por Scleroderma areolatum Ehrenb. en Estados Unidos. De igual manera Ott et al. (1975) describieron dos casos de intoxicación por la ingesta de S. verrucosum (Bull.) Pers. en Oaxaca. Recientemente Sato et al. (2020) documentaron un caso de intoxicación provocado por S. albidum Pat. \& Trab. en Japón, el paciente presentó síntomas semejantes a una intoxicación tipo muscarínica; sin embargo, no se sabe cuál es el compuesto tóxico en este hongo. Con relación a la toxicidad de Scleroderma en otros organismos, Ruiz-González et al. (2017) evaluaron la toxicidad de algunos hongos sobre Artemia franciscana Kellogg, entre ellos $S$. texense Berk., sus resultados indican que los extractos acuosos obtenidos de este hongo presentaron una toxicidad baja para ese crustáceo.

Además de lo anterior, algunas especies de Scleroderma han sido investigadas con fines médicos por sus propiedades antiinflamatorias y hemostáticas (Guzmán
1970; Liu 1984; Gurgel et al. 2008). Gong et al. (2005) citaron a S. polyrhizum (J.F. Gmel.) Pers. con usos en la medicina tradicional China para el tratamiento de hemostasis. González et al. (1983) presentaron un estudio sobre ácidos grasos y esteroides contenidos en los basidiomas de S. polyrhizum, de ellos el peróxido de ergosterol es considerado anticancerígeno y antiinflamatorio. Algunos otros compuestos químicos presentes en Scleroderma son derivados de ácidos pulvínicos; por ejemplo, la Norbadina A presente en otros miembros del orden Boletales (Rogers 2011; Velíŝek y Cejpek 2011), se sabe que este compuesto tiene ciertas propiedades protectoras a la radiactividad; sin embargo, en concentraciones altas resulta tóxica para ratones (Le Roux et al. 2012). Además de la Norbadina A, Winner et al. (2004) encontraron en Scleroderma citrinum un compuesto denominado sclerocitrina en grandes concentraciones, pero se desconoce su actividad biológica. En esta misma especie Rogers (2011) cita otros compuestos con propiedades antivirales y antibacterianas.

Los hongos juegan un papel relevante en la vida de diferentes grupos étnicos de Oaxaca, los cuales se caracterizan como micófilos debido al fuerte vínculo con estos organismos (Wasson y Wasson 1957). Esto se evidencia con los resultados de los trabajos llevados a cabo con los Zapotecos de la Sierra Norte (León-Avendaño 1995; Garibay-Orijel et al. 2006, 2007), Mazatecos y Mixes (Wasson y Wasson 1957; Vásquez-Dávila 2008), los Mixtecos de la Mixteca Alta (Hernández-Santiago et al. 2016; Aparicio-Aparicio 2019) y los Chinantecos de la Sierra Norte (López-García et al. 2017, 2020) por citar algunos. Aunque en Oaxaca se han llevado a cabo trabajos etnomicológicos; no se ha abarcado el complejo mosaico cultural que se presenta en el estado. Oaxaca alberga 16 grupos etnolingüisticos que a su vez presentan variantes culturales y lingüísticas dentro de cada grupo (Castillo-Cisneros 2006). En este sentido el conocimiento en torno a los hongos varía entre estos grupos entolingüisticos y regiones (Garibay-Orijel et al. 2006; Ruan-Soto et al. 2014). El objetivo del presente estudio fue documentar los saberes tradicionales del proceso de consumo de un hongo que pertenece a un género que se considera tóxico por algunos autores y comestible por otros, como una primera aproximación al conocimiento tradicional de la etnia zapoteca de la Sierra Sur de la localidad de Santiago Xochiltepec, Municipio de Santiago Textitlán, Sola de Vega, Oaxaca. 


\section{MATERIALES Y MÉTODOS}

Área de estudio

El trabajo se llevó a cabo en la comunidad de Santiago Xochiltepec perteneciente al Municipio de Santiago Textitlán, Distrito Político de Sola de Vega, de la Región Sierra Sur en el estado de Oaxaca. La comunidad se encuentra localizada en las coordenadas geográficas $\mathrm{N} 16^{\circ} 39^{\prime} 40.5^{\prime \prime}$ y O 97'14'49.7" a una altitud de 1787 m s.n.m. y se localiza a $152 \mathrm{~km}$ de la capital de Oaxaca (Figura 1). Tiene una temperatura entre los $12-24{ }^{\circ} \mathrm{C}$ y su precipitación anual varía entre 1500 y $2500 \mathrm{~mm}$. El clima es templado húmedo con abundantes lluvias en verano. La vegetación predominante es bosque de pino-encino. Esta comunidad está habitada por población indígena y mestiza cuya lengua materna es el zapoteco. La población está constituida de 485 mujeres y 433 hombres. La silvicultura es una de las principales actividades económicas en el municipio de Santiago Textitlán (Gómez-Martínez 2005; INEGI 2010).
Realización de encuestas

Los alumnos del Bachillerato Integral Comunitario $\mathrm{N}^{\circ}$ 9 en la localidad de Santiago Textitlán realizaron entrevistas informales y semiestructuradas (Bernard 2006) a 38 colaboradores clave de la localidad. Los temas abordados en las entrevistas incluyeron el uso de este hongo, los criterios para reconocerlo, morfología, formas de transmisión del conocimiento y la preparación culinaria. Los datos obtenidos de las entrevistas y observaciones se presentan de manera descriptiva en los resultados y se analizaron con base en la teoría fundamentada en los datos de Glaser y Strauss (Soneira 2006). También, se realizó un registro fotográfico del proceso de recolección y preparación culinaria del hongo para su consumo.

\section{Identificación de la especie}

Se efectuó observación participante durante la recolección de hongos, de esta manera una colección de la especie consumida por los entrevistados se re-

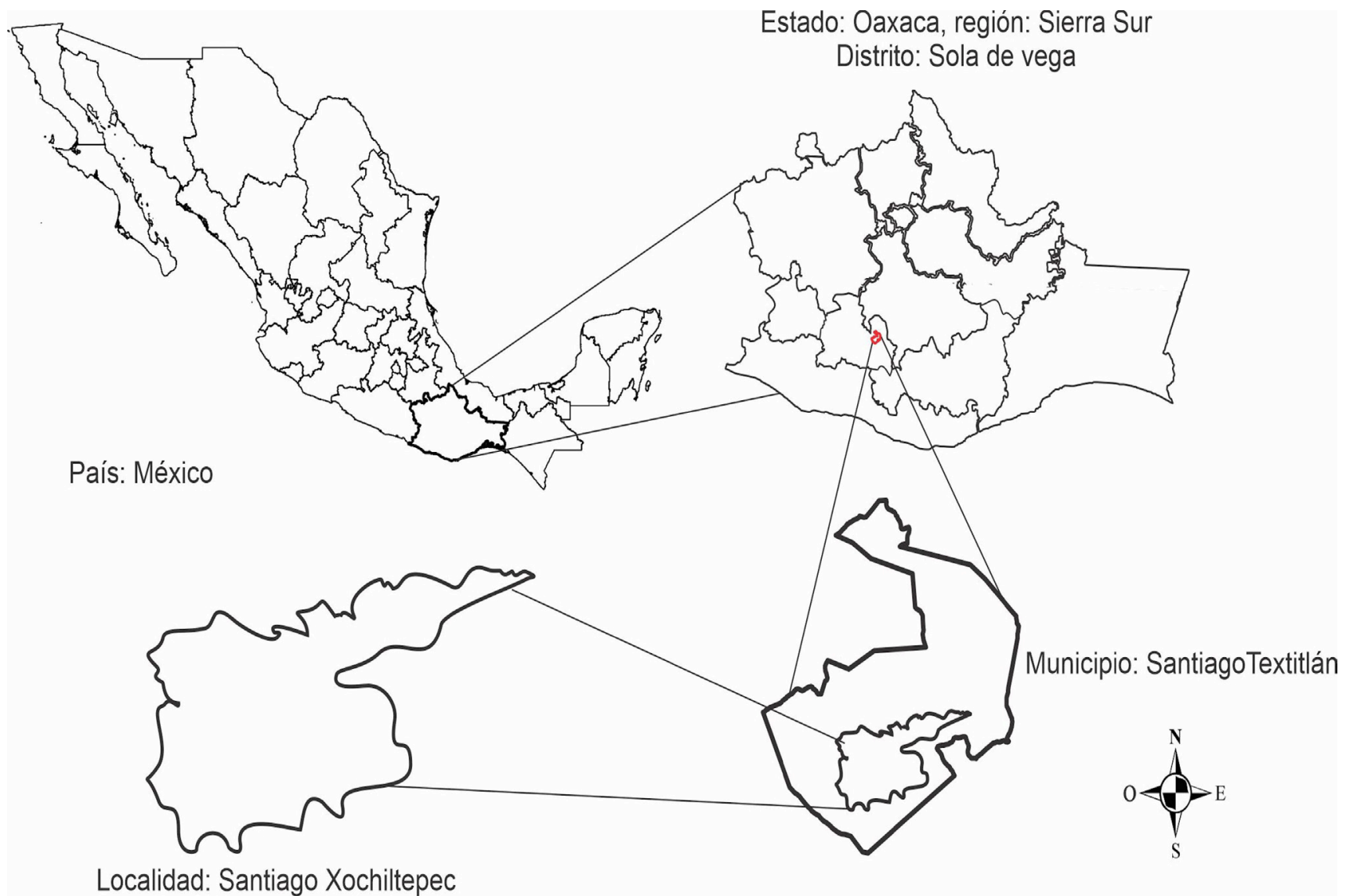

Figura 1. Ubicación geográfica del área de estudio. 
colectó de acuerdo con las técnicas empleadas en Micología (Cifuentes et al. 1986) y se depositó en la Colección de Hongos XAL e IBUG para su posterior identificación. El análisis microscópico se realizó bajo los métodos propuestos por Largent et al. (1977). Se elaboraron preparaciones con cortes a navaja del basidioma, que se montaron en solución de $\mathrm{KOH}$ al 5 $\%$, previamente rehidratados con alcohol al $70 \%$. Se tomaron 30 medidas de cada una de las estructuras de valor taxonómico. Para la identificación del ejemplar estudiado se consultó el trabajo de Guzmán et al. (2012).

\section{RESULTADOS}

\section{Estudio taxonómico}

A partir del estudio macro y micromorfológico del ejemplar recolectado se concluyó que corresponde a Scleroderma texense pues concuerda con la descripción de la especie (Guzmán et al. 2012). Esta especie pertenece a la sección Sclerangium y se distingue por el peridio escamoso a profusamente escamoso por agrietamiento, escamas planas de color blanquecino sobre un fondo amarillo paja; por las basidiosporas subreticuladas de 7-11 $\mu \mathrm{m}$ de diámetro y por las hifas fibuladas. En México es una especie que se distribuye en regiones subtropicales en donde dominan los bosques de Pinus-Quercus o bosques de niebla, se conoce de los estados de Chiapas, Guerrero, Hidalgo, Jalisco, Michoacán, Morelos, Oaxaca, Puebla, Sinaloa, Estado de México y Veracruz (Guzmán et al. 2012). El material estudiado se encontró solitario o gregario, creciendo en suelo cubierto con hojarasca o suelo desnudo. Subhipogeo cuando inmaduro a epigeo en la dehiscencia, creciendo en bosques de Pinus o Pinus-Quercus. Material estudiado: Oaxaca, México, Sola de Vega, Mpio. Santiago Xochiltepec, Santiago Textitlán, agosto 17, 2019, C. Pérez-Pacheco 01 (XAL, IBUG).

\section{Trabajo etnográfico}

Se presenta el segundo registro documentado de la comestibilidad del género Scleroderma en México y el primero en Oaxaca. Cabe señalar que según los entrevistados el hongo se consume únicamente en la comunidad de Santiago Xochiltepec y no en otras localidades del municipio. De las entrevistas realizadas a los pobladores de Santiago Xochiltepec se encontró que el hongo Scleroderma texense recibe el nombre común de "huevo de pato", "huevo de guajolota" o "guitarra". El 60 \% de los informantes conocen y consumen el hongo desde que son niños a la edad de 4 a 8 años, y es a través de sus abuelos o padres que obtienen el conocimiento y aprenden a reconocer esta especie. Para recolectar el hongo no existe una técnica particular, se realiza de la misma manera que con otros hongos, aunque se trata de un hongo subhipogeo este es fácil de ver en el bosque. En campo se seleccionan los ejemplares inmaduros y solamente con la mano se jalan y recolectan. El consumo de estos hongos se hace cuando están una fase intermedia de desarrollo, cuando la gleba es de color oscuro, pero no polvorienta y no presenta dehiscencia. Una vez maduro, éste se abre de manera esteliforme o subesteliforme dejando la gleba expuesta para ser dispersada por el viento o por la lluvia, cuando alcanza este estado ya no es comestible. Para consumirlo las personas los lavan para quitarles el exceso de tierra, después son asados en las brasas o sobre el comal, por último, retiran las partes cocidas del peridio y consumen la gleba inmadura combinándola con salsa de tomate o simplemente agregan sal para comerla con tortillas (Figura 2). Suelen comer entre 2 a 15 cuerpos fructíferos por familia, en algunas familias llegan a consumir de 20 a 30 esporomas dependiendo de la disponibilidad del hongo. Scleroderma texense no es de los hongos preferidos (por sabor) para los pobladores de la comunidad, en comparación con otras especies comestibles como Amanita del grupo caesarea, Boletus del grupo edulis, Cantharellus spp. o Ramaria spp. Sin embargo, tienen importancia cultural debido a que los esporomas son abundantes y fáciles de encontrar ya que crecen cerca de los caminos.

\section{DISCUSIÓN}

Los nombres comunes "huevo de guajolota" o "huevo de pato" hacen referencia a la forma globosa del hongo que recuerda a un huevo. Recibe también el nombre común de "guitarra" por el sonido que emite el peridio cuando se colocan los basidiomas en las brasas para asarse. Estos resultados coinciden con lo planteado por Berlin (1992) quién señala que los constituyentes lingüisticos de los nombres de los organismos a menudo aluden metafóricamente a características morfológicas, de comportamiento o ecológicas. El nombre tradicional "huevo" también es usado en la 

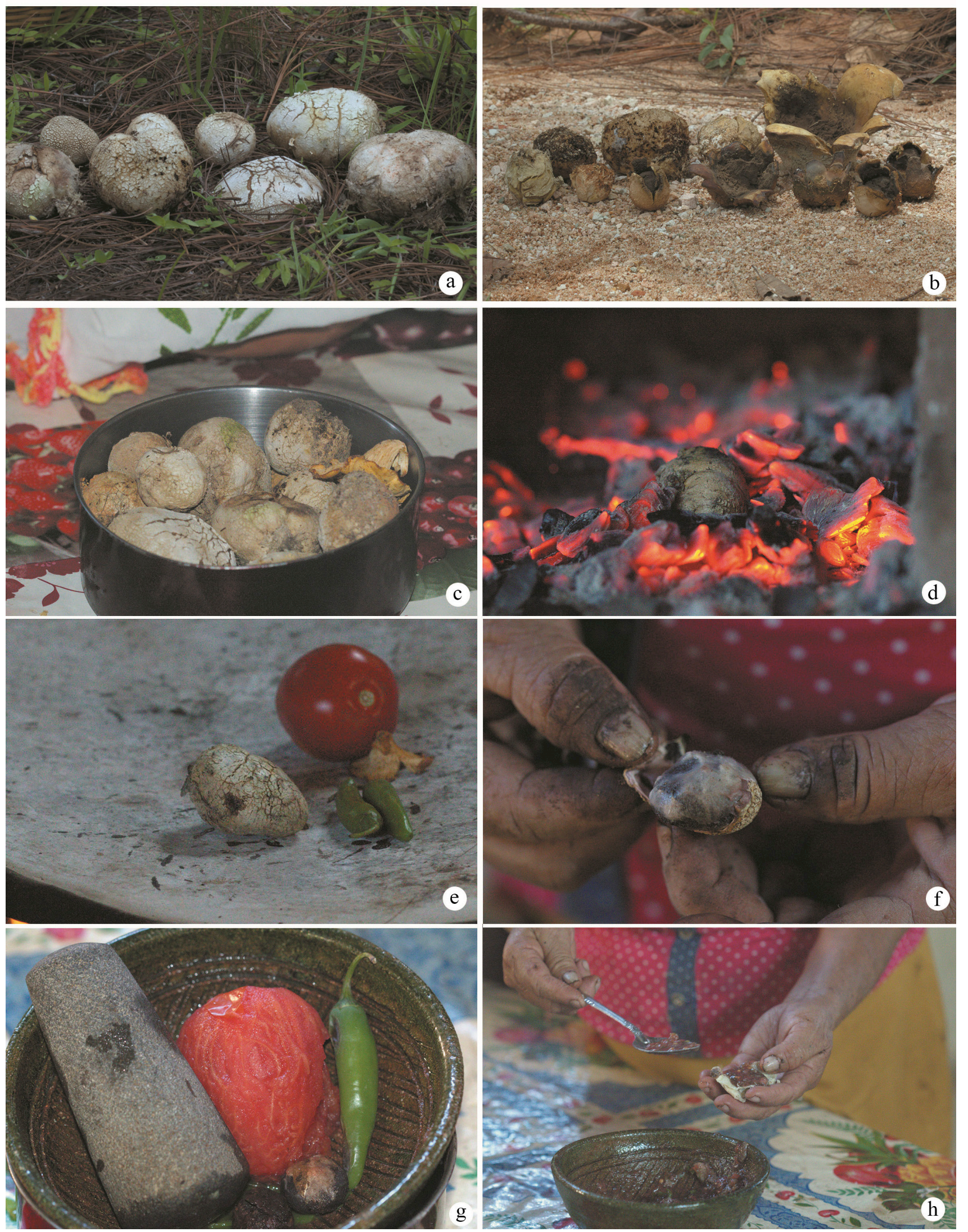

Figura 2. Fotografía en campo y preparación de Scleroderma texense. a) y b) esporomas de Scleroderma texense en diferentes estadios. c) esporomas recién lavados. d) y e) esporomas asados a las brasas y comal. f) esporomas asados con el peridio a punto de ser retirado. g) salsa de tomate con $\mathrm{S}$. texense. h) mujer a punto de consumir un esporoma. 
región de Molango, Hidalgo para referirse a Amanita tecomate Guzmán \& Ram.-Guill., Russula cessans A. Pearson y $R$. aff. pulchra Burl.; sin embargo, en estos hongos la coloración anaranjada rojiza que presentan estas tres especies está relacionado con el color de la yema de huevo de guajolota y no con la forma de los basidiomas (Jiménez-González et al. 2013).

Por otro lado, es interesante señalar que los hongos se consumen en estadios intermedios a maduros y no en estadios inmaduros como ocurre en otros géneros de esporomas gasteroides, por ejemplo, Lycoperdon Pers. y Calvatia (Guzmán 2008; Medina Arias et al. 2014). El mismo caso se presenta en Scleroderma yunnanense que se consume en estadios intermedios donde la gleba ya tiene un color gris obscuro, sin embargo, esta especie es consumida con mayor preferencia cuando está inmadura (Zhang et al. 2013; Wang et al. 2020).

El hallazgo de la comestibilidad de Scleroderma texense, los registros de otras especies comestibles dentro del género (De Ávila et al. 1980; Zhang et al. 2013) y el trabajo realizado por Ruiz-González et al. (2017), en donde S. texense no presentó toxicidad para Artemia franciscana, sugieren que dentro del género Scleroderma hay especies comestibles y tóxicas, como ocurre en otros géneros de hongos, por ejemplo, en Amanita Pers. y Agaricus L. (Karunarathna et al. 2016; Verma et al. 2020). Sin embargo, es necesario caracterizar los metabolitos secundarios presentes en las especies de Scleroderma, particularmente en esta especie para verificar la ausencia de toxinas. Además, se debe investigar la acción biológica de los compuestos, ya que en muchos casos se desconoce, por ejemplo, en la sclerocitrina encontrada en Scleroderma citrinum (Sato et al. 2020)

Finalmente, cabe señalar que el estado de Oaxaca es muy heterogéneo tanto biológicamente como culturalmente de tal forma que de una localidad a otra pueden variar las especies consumidas. Hasta la fecha sólo se ha documentado la comestibilidad de Scleroderma texense en esta comunidad ubicada Distrito de Sola de Vega en la región Sierra Sur de Oaxaca.

\section{AGRADECIMIENTOS}

Los autores agradecen a los habitantes de la comunidad de Santiago Xochiltepec por compartir sus conocimientos y a los alumnos del Bachillerato Integral Comunitario No. 9 de esta localidad, por aplicar las entrevistas.

\section{LITERATURA CITADA}

Aparicio-Aparicio JC. 2019. Taxonomía mixteca y uso de los hongos en San Miguel El Grande, Oaxaca, México. Revista Etnobiología $17,18-30$

Berlin B. 1992. Ethnobiological classification: principles of categorization of plants and animals in traditional societies. Princeton University Press, New Jersey.

Bernard HR. 2006. Research methods in anthropology: qualitative and quantitative approaches. Altamira Press, Lanham, Maryland.

Castillo-Cisneros MC. 2006. Tacuates. Comisión Nacional para el Desarrollo de los Pueblos Indígenas, México, D.F.

Cifuentes J, Villegas M, Pérez-Ramírez L. 1986. Hongos. In: Lot A, Chiang $F$ (eds.) Manual de herbario. Consejo Nacional de la Flora de México A.C., México, D.F. 55-64.

Coccia M, Migliozzi Y, Lavorato C. 1990. Studio sul genere Scleroderma Persoon. Bolletino dell'Associazione Micologica ed Ecologica Romana 20, 3-59.

De Ávila AB, Welden AL, Guzmán G. 1980. Notes on the ethnomycology of Hueyapan, Morelos, México. Journal of Ethnopharmacology 2, 311-321. https://doi.org/10.1016/S03788741(80)81013-0

Garibay-Orijel R, Cifuentes J, Estrada-Torres A, Caballero J. 2006. People using macro-fungal diversity in Oaxaca, Mexico. Fungal Diversity 21, 41-67.

Garibay-Orijel R, Caballero J, Estrada-Torres A, Cifuentes J. 2007. Understanding cultural significance, the edible mushrooms case. Journal of Ethnobiology and Ethnomedicine 3, 4. https:// doi:10.1186/1746-4269-3-4

Gómez-Martínez E. 2005. Zapotecos de la Sierra Sur de Oaxaca. Proyecto etnografía contemporánea de los pueblos indígenas de México. CDI, Oaxaca.

Gong XL, Zeng RS, Luo SM. 2005. Chemical constituents from the fruit bodies of Scleroderma polyrhizum. Natural Product Research and Development 17, 431-433.

González AG, Barrera JB, Marante FJT. 1983. The steroids and fatty acids of the Basidiomycete Scleroderma polyrhizum. Phytochemistry 22, 1049-1050. https://doi.org/10.1016/0031. 9422(83)85062-6

Gurgel FE, Silva BDB, Baseia IG. 2008. New records of Scleroderma from Northeastern Brazil. Mycotaxon 105, 399-405.

Guzmán G. 1970. Monografía del género Scleroderma Pers. emend. Fr. (Fungi-Basidiomycetes). Darwiniana 16, 233-401.

Guzmán G. 1977. Identificación de los hongos comestibles, venenosos y destructores de la madera. Limusa, México, D.F.

Guzmán G. 1980. Las intoxicaciones producidas por los hongos. Ciencia y Desarrollo 32, 129-134.

Guzmán G. 2008. Hongos de parques y jardines y sus relaciones con la gente. Secretaria de Educación de Veracruz, Xalapa.

Guzmán G, Cortés-Pérez A, Guzmán-Dávalos L, Ramírez-Guillén F, Sánchez-Jácome R. 2013. An emendation of Scleroderma, new records, and review of the known species in Mexico. Revista Mexicana de Biodiversidad 84, 173-191. https://doi. org/10.7550/rmb.31979

He MQ, Zhao RL, Hyde KD, Begerow D, Kemler M, Yurkov A, McKenzie EHC, Raspé O, Kakishima M, Sánchez-Ramírez S, Vellinga EC, Halling R, Papp V, Zmitrovich IV, Buyck B, Ertz D, Wijayawardene 
NN, Cui BK, Schoutteten N, Liu XZ, Li TH, Yao YJ, Zhu XY, Liu AQ, Li GJ, Zhang MZ, Ling ZL, Cao B, Antonín V, Boekhout T, da Silva BDB, De Crop E, Decock C, Dima B, Dutta AK, Fell JW, Geml J, Ghobad-Nejhad M, Giachini AJ, Gibertoni TB, Gorjón SP, Haelewaters D, He SH, Hodkinson BP, Horak E, Hoshino T, Justo A, Lim YW, Menolli N Jr, Mešić A, Moncalvo JM, Mueller GM, Nagy LG, Nilsson RH, Noordeloos M, Nuytinck J, Orihara T, Ratchadawan C, RajchenbergM, Silva-Filho AGS, Sulzbacher MA, Tkalčec Z, Valenzuela R, Verbeken A, Vizzini A, Wartchow F, Wei TZ, Weiß M, Zhao CL, Kirk PM. 2019. Notes, outline and divergence times of Basidiomycota. Fungal Diversity 99,105-367. https://doi.org/10.1007/s13225-019-00435-4

Hernández-Santiago F, Pérez-Moreno J, Xoconostle-Cázares B, Almaráz-Suárez JJ, Ojeda-Trejo E, Mata G, Díaz-Aguilar I. 2016. Traditional knowledge and use of wild mushrooms by Mixtecs or Nuu savi, the people of the rain, from southeastern Mexico. Journal of Ethnobiology and Ethnomedicine 12(35), 1-22. https://doi.org/10.1186/s13002-016-0108-9

INEGI, 2010. Instituto Nacional de Estadística y Geografía. Marco Geoestadístico Municipal. Prontuario de información geográfica municipal de los Estados Unidos Mexicanos. Santiago Textitlán, Oaxaca Clave Geoestadística 20491.

Jiménez-González M, Romero-Bautista L, Villavicencio-Nieto MA, Pérez-Escandón BE. 2013. Los hongos comestibles de la región de Molango de Escamilla, Hidalgo, México. In: Pulido-Flores G, Monks S. (eds.) Estudios científicos en el estado de Hidalgo y zonas aledañas 11. Zea Books, Lincoln. 69-82.

Karunarathna S, Chen J, Mortimer P, Xu J, Zhao RL, Callac P, Hyde KD. 2016. A review of genus Agaricus in tropical and humid subtropical regions of Asia. Mycosphere 7, 417-439. https://doi. org/10.5943/mycosphere/7/4/3

Kirk PM, Cannon PF, Minter DW, Stalpers JA. 2008. Dictionary of the Fungi. CABI, Wallingford.

Largent D, Johnson D, Watling R. 1977. How to identify mushrooms to genus III: microscopic features. Mad River Press, Eureka.

León-Avendaño H. 1995. Aprovechamiento y perspectivas del cultivo de hongos comestibles silvestres de la Sierra Juárez de Oaxaca. In: Vázquez-Dávila DH (ed.) La tecnología agrícola tradicional. Sociedad y Naturaleza en Oaxaca-Instituto Indigenista Interamericano-Consejo Nacional de Ciencia y Tecnología, Oaxaca. 114-138.

Le Roux A, Josset E, Benzina S, Nadal B, Desage-El Murr M, Heurtaux B, Taran F, Denis JM, Gall TL, Meunier S, Bischoff P. 2012. Evaluation of the radioprotective potential of the polyphenol Norbadione A. Letters in Drug Design \& Discovery 9, 48-53. https://doi.org/10.2174/157018012798192900

Liu B. 1984. The Gasteromycetes of China. Beihefte Nova Hedwigia $76,1-235$.

López-García A, Jiménez-Ruiz M, Pérez-Moreno J. 2017. Vocablos relacionados con el recurso micológico en el idioma de la cultura chinanteca de la Sierra Norte del estado de Oaxaca, México. Scientia Fungorum 46, 9-18. https://doi.org/10.33885/ sf.2017.46.1171

López-García A, Pérez-Moreno J, Jiménez-Ruiz M, Ojeda-Trejo E, Delgadillo-Martínez J, Hernández-Santiago F. 2020. Conocimiento tradicional de hongos de importancia biocultural en siete comunidades de la región chinanteca del estado de Oaxaca, México. Scientia Fungorum 50, e1280. https://doi.org/10.33885/ sf.2020.50.1280

Medina Arias FG, Andrade Gallegos RH, Sánchez JE. 2014. Ethnomycology in the "Tacaná Volcano Biosphere Reserve", Chiapas, Mexico. Proceedings of the 8th International Conference on Mushroom Biology and Mushroom Products ((ICMBMP8), New Delhi. 98-107.

Murrill WA.,1910. Ilustrations of fungi: V. Mycologia 1, 1-6.

Ott J, Guzmán G, Romano J, Díaz JL. 1975. Nuevos datos sobre los supuestos licoperdáceos psicotrópicos y dos casos de intoxicación provocados por hongos del género Scleroderma. Boletín Sociedad Mexicana de Micología 9, 67-76.

Rogers R. 2011. The fungal pharmacy: The complete guide to medicinal mushrooms \& lichens of North America. North Atlantic Books, Berkeley.

Ruan-Soto F, Caballero-Nieto J, Cifuentes J, Garibay-Orijel R. 2014. Micofilia y Micofobia: revisión de los conceptos, su reinterpretación e indicadores para su evaluación. In: Moreno Fuentes A, Garibay-Orijel R (eds.) La micología en Mexico. Estado del arte. Red de Etnoecología y Patrimonio Biocultural (CONACyT), Universidad Autónoma del Estado de Hidalgo, Instituto de Biología (UNAM), Sociedad Mexicana de Micología, Asociación Etnobiológica Mexicana, Grupo Interdisciplinario para el Desarrollo de la Etnomicología en México, Sociedad Latinoamericana de Etnobiología. México, D.F. 17-30.

Ruiz-González LE, Vázquez-Zea JA, Vega-Villasante F, Guzmán-Dávalos L, Guerrero-Galván SR. 2017. Evaluación del efecto tóxico de hongos Basidiomycota en la eclosión de quistes de Artemia franciscana. Revista Iberoamericana de Micología 34, 220-224. https://doi.org/10.1016/j.riam.2017.03.007

Sato Y, Tomonari H, Kaneko Y, Yo K. 2020. Mushroom poisoning with Scleroderma albidum: a case report with review of the literature. Acute Medicine \& Surgery 7, e460. https://doi.org/10.1002/ ams 2.460

Sebek S. 1953. Monograph of the Central European species of the genus Scleroderma Pers. Sydowia 7, 158-190.

Soneira AJ. 2006. La teoría fundamentada en los datos (grounded theory) de Glaser y Strauss. In: Vasilachis I (ed.) Estrategias de investigación cualitativa. Gedisa, Barcelona. 153-173.

Stevenson JA, Benjamin CR. 1961. Scleroderma poisoning. Mycologia 53, 438-439. http://dx.doi.org/10.2307/3756589

Vásquez-Dávila MA. 2008. Atlas etnomicológico de Oaxaca, México: avances de una década. Etnobiología 6, 85-87.

Velíŝek J, Cejpek K. 2011. Pigments of higher fungi: A review. Czech Journal of Food Sciences 29, 87-102.

Verma R, Pandro V, Rao G. 2020. Two new records of gilled mushrooms of the genus Amanita (Agaricales: Amanitaceae) from India. Journal of Threatened Taxa 12: 15194-15200. http://dx.doi. org/10.11609/jott.4822.12.1.15194-15200

Wang Y, Yu FQ, Zhang C, Liu C, Yang M, Li S. 2020. Edible ectomycorrhizal fungi and their cultivation in China. In: Pérez-Moreno J, Guerin-Laguette A, Flores Arzú R, Yu F-Q (eds.) Mushrooms, humans and nature in a changing world. Springer, Cham. Switzerland. 31-60. https://doi.org/10.1007/978-3-030-37378-8 2 
Wasson VP, Wasson RG. 1957. Mushrooms, Russia and history. Pantheon, New York.

Winner M, Giménez A, Schmidt H, Sontang B, Steffan B, Steglich W. 2004. Unusual pulvinic acid dimers from the common fungi Scleroderma citrinum (common earthball) and Chalciporus piperatus (peppery bolete). Angewandte Chemie 43, 18831886. https://doi.org/10.1002/anie.2003352529

Zhang C, Xu X-E, Liu J, He M, Wang W,Wang Y, Ji K. 2013. Scleroderma yunnanense, a new species from South China. Mycotaxon 125, 193-200. https://doi.org/10.5248/125.193 